\title{
Increased FGF19 copy number is frequently detected in hepatocellular carcinoma with a complete response after sorafenib treatment
}

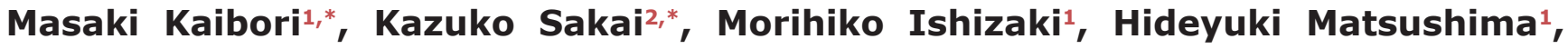 \\ Marco A. De Velasco ${ }^{2}$, Kosuke Matsui ${ }^{1}$, Hiroya Iida ${ }^{1}$, Hiroaki Kitade ${ }^{1}$, A-Hon \\ Kwon ${ }^{1}$, Hiroaki Nagano ${ }^{3}$, Hiroshi Wada ${ }^{3}$, Seiji Haji ${ }^{4}$, Tadashi Tsukamoto5, Akishige \\ Kanazawa $^{5}$, Yutaka Takeda ${ }^{6}$, Shigekazu Takemura7, Shoji Kubo7, Kazuto Nishio² \\ ${ }^{1}$ Department of Surgery, Hirakata Hospital, Kansai Medical University, Hirakata, Osaka, 573-1010, Japan \\ ${ }^{2}$ Department of Genome Biology, Kinki University Faculty of Medicine, Osakasayama, Osaka, 589-8511, Japan \\ ${ }^{3}$ Department of Gastroenterological Surgery, Osaka University Graduate School of Medicine, Osaka, 565-0871, Japan \\ ${ }^{4}$ Department of Surgery, Kinki University Faculty of Medicine, Osakasayama, Osaka, 589-8511, Japan \\ ${ }^{5}$ Department of Hepato-Biliary-Pancreatic Surgery, Osaka City General Hospital, Miyakojima, Osaka, 534-0024, Japan \\ ${ }^{6}$ Department of Surgery, Kansai Rosai Hospital, Amagasaki, Hyogo, 660-8511, Japan \\ ${ }^{7}$ Department of Hepato-Biliary-Pancreatic Surgery, Osaka City University Graduate School of Medicine, Osaka, 558-8585, \\ Japan \\ *These authors have contributed equally to this work \\ Correspondence to: Kazuto Nishio, email: knishio@med.kindai.ac.jp \\ Keywords: FGF19, sorafenib, copy number gain, hepatocellular carcinoma \\ Received: November 04, $2015 \quad$ Accepted: May 17, $2016 \quad$ Published: June 15, 2016
}

\section{ABSTRACT}

The multi-kinase inhibitor sorafenib is clinically approved for the treatment of patients with advanced hepatocellular carcinoma (HCC). We previously reported that fibroblast growth factor 3 and 4 (FGF3/FGF4) amplification is a predictor of a response to sorafenib. This study aims to analyze the relationship between FGF-FGF receptor (FGFR) genetic alterations and the response to sorafenib. Formalin-fixed, paraffinembedded tissue specimens from HCC patients who had achieved a complete response $(C R, N=6)$ or non-CR $(N=39)$ to sorafenib were collected and were examined for $F G F-$ FGFR gene alterations using next generation sequencing and copy number assay. FGFR mutations were detected in 5 of $45(11.1 \%)$ cases. There was no significant association between FGFR mutation status and the response to sorafenib. We detected no increase in the FGF3/FGF4 copy number in CR cases. An FGF19 copy number gain was detected more frequently among $C R$ cases $(2 / 6,33.3 \%)$ than among non-CR cases $(2 / 39,5.1 \%)(P=0.024$, Chi-squared test $)$. In conclusion, a copy number gain for FGF19 may be a predictor of a response to sorafenib, in addition to FGF3/FGF4 amplification.

\section{INTRODUCTION}

Hepatocellular carcinoma (HCC) is the most common primary malignancy of the liver [1]. Several reports have suggested that hepatocarcinogenesis involves multiple molecular pathways involving the accumulation of genetic and epigenetic alterations, including copy number aberration and gene mutations [2] [3] [4]. Sorafenib is a multi-targeted kinase inhibitor that has a potent antitumor activity in several preclinical models [5]. Sorafenib is now used as standard therapy for advanced HCC [6] [7]. However, a complete response is very rare, and the response rate is low (between $0.7 \%$ and 3.3\%) [6] [7]. Nevertheless, complete responses were observed in patients with advanced HCC after shortterm treatment with sorafenib [8] [9] [10]. We previously reported the clinical and molecular backgrounds of 13 responders to sorafenib with significant tumor shrinkage 
in a retrospective study [11]. A comparative genomic hybridization analysis using one frozen HCC sample from a responder demonstrated that the $11 \mathrm{q} 13$ region, a rare amplicon in HCC including the loci for FGF3 and $F G F 4$, was highly amplified. However, the mechanisms responsible for the responses to sorafenib in the remaining cases without $F G F 3 / F G F 4$ amplification remain unclear.

In this study, we further analyze molecular alterations on the FGF-FGFR signal pathway using the different sample cohort from that of our previous report [11]. The FGF pathway is aberrantly activated through a variety of genetic alterations in many types of cancers [12]. Recently, several studies have reported that gene mutation or amplification in $F G F / F G F R$ predict for sensitivity of FGFR inhibitors. FGFR1, FGFR2 or $F G F 19$ gene amplifications were reported as potential biomarkers of a selective FGFR inhibitor [13] [14]. Thus, a genetic alteration in $F G F / F G F R$ is considered to be a potential biomarker for effective FGFR inhibition. Here, we focused on $F G F / F G F R$ gene alterations to elucidate other mechanisms related to sorafenib response because sorafenib potentially inhibits FGFR kinase activity [5] [15]. In the current study, we collected responder cases (PR and CR) to sorafenib and conducted a case-control study with a retrospective design in order to explore the association between the efficacy of sorafenib and gene alterations, including copy number changes and mutations, in FGF-FGFR signals.

\section{RESULTS}

\section{Baseline characteristics}

The characteristics of the patients are summarized in Table 1. The tumor samples of complete response $(\mathrm{CR})(\mathrm{N}=6)$ and partial response $(\mathrm{PR})(\mathrm{N}=4)$ cases were collected. Samples of stable disease (SD) $(\mathrm{N}=13)$ and progress disease (PD) $(\mathrm{N}=22)$ cases were also collected as control. Patients with recurrence after curative surgery were treated with sorafenib. The median time from surgery to initiation of sorafenib therapy of CR, PR, SD, and $\mathrm{PD}$ cases were 35.8 months (range, 9.8-82.1), 32.5 months (range, 20.4-57.8), 9.6 months (range, 1.4-66.3), and 1.4 months (range, 0.4-56.9), respectively. Three of the six CR cases (case no. CR4, CR5, and CR6) received transcatheter arterial chemoembolization (TACE) before starting sorafenib treatment. Two cases (case no. CR1 and $\mathrm{CR} 2$ ) received TACE before and during treatment with sorafenib. One case (case no. CR3) received TACE/ hepatic arterial infusion chemotherapy (HAIC) before and during treatment with sorafenib. Three PR cases (case no. PR1, PR2, and PR4) received TACE, TACE/radiation, and TACE/radiofrequency ablation (RFA) before sorafenib treatment, respectively. One PR case (case no. PR3) received only sorafenib treatment. Ten of the $13 \mathrm{SD}$ cases received TACE before starting sorafenib treatment. Eight of 22 PD cases received TACE before starting sorafenib treatment. The remaining $17 \mathrm{SD}$ and $\mathrm{PD}$ cases received only sorafenib treatment. Median time from first drug administration to the date of the first clinical complete and partial response was 4.0 months (range; 1.5-33.7) and 2.2 months (range; 1.7-14.2), respectively.

The majority of the cases exhibited Child-Plug class A, single tumor and moderate differentiation. Advanced stages (TNM stages III and IV) were classified in nonresponder (SD and $\mathrm{PD})$ cases.

\section{FGFR mutation analysis in complete or partial responders}

Genomic DNA was subjected to FGFR1-4 mutation analysis. We screened all exons of FGFRs (FGFR1, FGFR2, FGFR3, and FGFR4) in 45 cases. We identified FGFR2 mutations in $2(4.4 \%)$, FGFR3 mutations in $2(4.4 \%)$, and FGFR1 and FGFR4 mutations in 1 each $(2.2 \%)$ (Supplementary Table S1). One CR case exhibited an FGFR1 $1^{5602 F}$ mutation located in the kinase domain. Another PR case exhibited both FGFR2 $2^{M 538 I}$ and FGFR $4^{G 665 E}$ mutations, also located in the kinase domain. No mutations were found in the remaining eight cases. FGFR3 mutations were detected only in SD or PD cases. In liver cancer, no mutation of the FGFR1 gene was found on the TCGA database (cBioPortal database). FGFR $1^{S 602 F}$ was reported in only one case of melanomaand FGFR2, and FGFR4 mutations were found in 4/231 (1.7\%) and $1 / 231(0.4 \%)$ liver cancer samples, respectively. Neither FGFR2 $2^{M 538}$ nor FGFR4 $4^{G 665 E}$ mutations were found in any of the studies available on the cBioPortal database. No experimental reports have discussed the functional changes associated with FGFR1 $1^{S 602 F}, F G F R 2^{M 538 I}$, or FGFR $4^{G 665 E}$ mutations. We retrieved functional impact scores from the Mutation Assessor database. A higher score of predicted functional impact indicates a higher likelihood of a functional mutation (i.e., a driver mutation). A low predicted functional impact of the FGFR $1^{S 602 F}$ mutation was obtained. On the other hand, FGFR2 $2^{\text {M538I }}$ or FGFR $4^{G 665 E}$ mutations were projected to produce a medium functional impact. We analyzed the association between FGFR mutation status with sorafenib response and the following variables: sex, virus infection, ChildPlug class, tumor size, and number of tumors. As shown in Table 2, there was no significant association between mutation status and the clinical variables.

\section{Copy number alterations in $F G F$ and $F G F R$ genes}

Copy number alterations in the tumor samples were analyzed using TaqMan chemistry. We screened the copy numbers of FGF3, FGF4, FGF19, FGFR1, FGFR2, FGFR3, and FGFR4 in CR and PR samples. In this assay, a cut-off value for copy number gain was set 
Table 1: Patient characteristics $(\mathrm{N}=45)$

\begin{tabular}{|c|c|c|c|c|}
\hline & $\mathrm{CR}(\mathrm{N}=6)$ & $\operatorname{PR}(\mathrm{N}=4)$ & $\mathrm{SD}(\mathrm{N}=13)$ & PD $(\mathrm{N}=22)$ \\
\hline \multicolumn{5}{|l|}{ Age (years) } \\
\hline Median (range) & $76.5(70-80)$ & $71.0(67-83)$ & $67.0(57-86)$ & $68.0(45-82)$ \\
\hline \multicolumn{5}{|l|}{ Sex } \\
\hline Male (\%) & $4(66.7)$ & $3(75.0)$ & $12(92.3)$ & $16(72.7)$ \\
\hline Female (\%) & $2(33.3)$ & $1(25.0)$ & $1(7.7)$ & $6(27.3)$ \\
\hline \multicolumn{5}{|l|}{ Hepatitis virus status } \\
\hline $\operatorname{HBV}(\%)$ & $1(16.7)$ & $0(0)$ & $1(7.7)$ & $2(9.1)$ \\
\hline $\operatorname{HCV}(\%)$ & $2(33.3)$ & $2(50.0)$ & $6(46.2)$ & $9(40.9)$ \\
\hline $\mathrm{HBV} / \mathrm{HCV}(\%)$ & $0(0)$ & $0(0)$ & $0(0)$ & $3(13.6)$ \\
\hline NBNC (\%) & $3(50.0)$ & $2(50.0)$ & $6(46.2)$ & $8(36.4)$ \\
\hline \multicolumn{5}{|l|}{ Child-Plug class } \\
\hline $\mathrm{A}(\%)$ & $6(100)$ & $4(100)$ & $12(92.3)$ & $21(95.5)$ \\
\hline B $(\%)$ & $0(0)$ & $0(0)$ & $1(7.7)$ & $1(4.5)$ \\
\hline \multicolumn{5}{|l|}{ Tumor size (cm) } \\
\hline Median (range) & $3.7(2.0-7.0)$ & $2.9(2.5-4.5)$ & $7.0(1.5-25.0)$ & $9.0(1.4-65.0)$ \\
\hline \multicolumn{5}{|l|}{ Number of tumors } \\
\hline Single (\%) & $6(100)$ & $3(75.0)$ & $9(69.2)$ & $12(54.5)$ \\
\hline Multiple (\%) & $0(0)$ & $1(25.0)$ & $4(30.8)$ & $10(45.5)$ \\
\hline \multicolumn{5}{|l|}{ Histology } \\
\hline Well differentiated (\%) & $1(16.7)$ & $0(0)$ & $2(15.4)$ & $1(4.5)$ \\
\hline Moderately differentiated (\%) & $5(83.3)$ & $2(50.0)$ & $11(84.6)$ & $18(81.8)$ \\
\hline Poorly differentiated (\%) & $0(0)$ & $2(50.0)$ & $0(0)$ & $3(13.6)$ \\
\hline \multicolumn{5}{|l|}{ TNM stage } \\
\hline $\mathrm{I}(\%)$ & $4(66.7)$ & $3(75.0)$ & $1(7.7)$ & $1(4.5)$ \\
\hline II $(\%)$ & $2(33.3)$ & $0(0)$ & $8(61.5)$ & $8(36.4)$ \\
\hline III (\%) & $0(0)$ & $1(25.0)$ & $2(15.4)$ & $11(50.0)$ \\
\hline IV $(\%)$ & $0(0)$ & $0(0)$ & $2(15.4)$ & $2(9.1)$ \\
\hline
\end{tabular}

Abbreviations: $\mathrm{CR}$, complete response; $\mathrm{PR}$, partial response; $\mathrm{SD}$, stable disease; $\mathrm{PD}$, progress disease; HBV, hepatitis B; $\mathrm{HCV}$, hepatitis $\mathrm{C}$; NBNC, non-hepatitis B and $-\mathrm{C}$.

at 5.00 for each gene. Increased copy numbers of FGF19 were detected in two CR $(2 / 6,33.3 \%)$ cases. No copy number gains were detected for $F G F 3, F G F 4, F G F R 1$, FGFR2, FGFR3, or FGFR4. To investigate whether an $F G F 19$ copy number gain was associated with a response to sorafenib, we analyzed additional tumor specimens from 35 patients who did not respond to sorafenib. Thirteen patients had SD and 22 had PD, as evaluated after sorafenib treatment. Increased copy numbers of FGF19 were detected in 2/35 (5.7\%) cases: 1/13 (7.7\%) among
SD cases and $1 / 22(4.5 \%)$ among PD cases. $F G F 3 / F G F 4$ copy numbers were concomitantly increased with $F G F 19$ in one of each case. No copy number gains were detected for FGFR1, FGFR2, FGFR3 or FGFR4. Additionally, increased FGF19 copy numbers and FGFR mutations were mutually exclusive. We compared the frequency of FGF 19 copy number gain in these cases. An FGF19 copy number gain was detected more frequently among CR cases than among non-CR cases $(P=0.024$, Chisquared test) (Table 2 and Supplementary Table S1). As 
Table 2: Associations of FGF19 copy number alterations, FGFR mutations, and clinical variables

\begin{tabular}{|c|c|c|c|c|c|c|c|}
\hline & \multirow[t]{2}{*}{$\mathbf{N}$} & \multicolumn{2}{|c|}{ FGF19 copy number gain } & \multirow[t]{2}{*}{$\boldsymbol{P}$} & \multicolumn{2}{|c|}{ FGFR mutation ${ }^{\text {a) }}$} & \multirow[t]{2}{*}{$\boldsymbol{P}$} \\
\hline & & $\begin{array}{c}\text { Negative } \\
\text { cases No. }(\%)\end{array}$ & $\begin{array}{c}\text { Positive } \\
\text { cases No. } \\
(\%)\end{array}$ & & $\begin{array}{c}\text { Negative } \\
\text { cases No. } \\
(\%)\end{array}$ & $\begin{array}{c}\text { Positive } \\
\text { cases No. } \\
(\%)\end{array}$ & \\
\hline \multicolumn{8}{|c|}{ Response to sorafenib (RECIST ver1.1) } \\
\hline $\mathrm{CR}$ & 6 & $4(66.7)$ & $2(33.3)$ & & $5(83.3)$ & $1(16.7)$ & \\
\hline $\begin{array}{l}\text { Non-CR (PR, } \\
\text { SD, PD) }\end{array}$ & 39 & $37(94.9)$ & $2(5.1)$ & $0.024 *$ & $35(89.7)$ & $4(10.3)$ & 0.642 \\
\hline \multicolumn{8}{|l|}{ Sex } \\
\hline Male $(\%)$ & 35 & $32(91.4)$ & $3(8.6)$ & & $31(88.6)$ & $4(11.4)$ & \\
\hline Female $(\%)$ & 10 & $9(90.0)$ & $1(10.0)$ & 0.889 & $9(90.0)$ & $1(10.0)$ & 0.899 \\
\hline \multicolumn{8}{|l|}{ Hepatitis virus status } \\
\hline $\begin{array}{l}\mathrm{HBV} \text { or } \mathrm{HCV} \\
\text { positive }(\%)\end{array}$ & 26 & $23(88.5)$ & $3(11.5)$ & & $23(88.5)$ & $3(11.5)$ & \\
\hline NBNC $(\%)$ & 19 & $18(94.7)$ & $1(5.3)$ & 0.465 & $17(89.5)$ & $2(10.5)$ & 0.915 \\
\hline \multicolumn{8}{|l|}{ Child-Plug class } \\
\hline$A(\%)$ & 43 & $39(90.7)$ & $4(9.3)$ & & $38(88.4)$ & $5(11.6)$ & \\
\hline $\mathrm{B}(\%)$ & 2 & $2(100.0)$ & $0(0.0)$ & 0.651 & $2(100.0)$ & $0(0.0)$ & 0.609 \\
\hline \multicolumn{8}{|l|}{ Tumor size (cm) } \\
\hline Median (range) & & $7.0(1.1-65.0)$ & $3.5(2.8-6.0)$ & 0.123 & $5.8(1.4-65.0)$ & $9.0(2.5-11.0)$ & 0.594 \\
\hline \multicolumn{8}{|l|}{ Number of tumors } \\
\hline Single (\%) & 30 & $27(90.0)$ & $3(10.0)$ & & $26(86.7)$ & $4(13.3)$ & \\
\hline Multiple (\%) & 15 & $14(93.3)$ & $1(6.7)$ & 0.711 & $14(93.3)$ & $1(6.7)$ & 0.502 \\
\hline \multicolumn{8}{|l|}{ Histology } \\
\hline $\begin{array}{l}\text { Well } \\
\text { differentiated }(\%)\end{array}$ & 4 & $4(100.0)$ & $0(0.0)$ & & $3(75.0)$ & $1(25.0)$ & \\
\hline $\begin{array}{l}\text { Moderately } \\
\text { differentiated (\%) }\end{array}$ & 36 & $33(91.7)$ & $3(8.3)$ & & $34(94.4)$ & $2(5.6)$ & \\
\hline $\begin{array}{l}\text { Poorly } \\
\text { differentiated (\%) }\end{array}$ & 5 & $4(80.0)$ & $1(20.0)$ & ND & $3(60.0)$ & $2(40.0)$ & ND \\
\hline \multicolumn{8}{|l|}{ TNM stage } \\
\hline I $(\%)$ & 9 & $7(77.8)$ & $2(22.2)$ & & 8 (88.9) & $1(11.1)$ & \\
\hline II (\%) & 18 & $16(88.9)$ & $2(11.1)$ & & $15(83.3)$ & $3(16.7)$ & \\
\hline III (\%) & 14 & $14(100.0)$ & $0(0.0)$ & & $13(92.9)$ & $1(7.1)$ & \\
\hline IV (\%) & 4 & $4(100.0)$ & $0(0.0)$ & ND & $4(100.0)$ & $0(0.0)$ & ND \\
\hline
\end{tabular}

Abbreviations: CR, complete response; PR, partial response; SD, stable disease; PD, progress disease; ND, not determined. ${ }^{*} P<0.05$ (Chi-squared test)

a) Mutations in FGFR1, FGFR2, FGFR3, and FGFR4

shown in Table 2, there was no significant relationship between increased FGF19 copy number and sex, virus infection, Child-Plug class, tumor size, and number of tumors. We also confirmed the frequency of FGF19 copy number alterations using cBioPortal for Cancer Genomics (Figure 1). The frequency of FGF19 amplification in a TCGA liver cancer dataset (15/193, 7.8\%) was consistent with the data obtained from the 35 non-responders. 
FGF19 gene amplification was considered to be a possible predictive biomarker for the efficacy of sorafenib in patients with HCC.

\section{DISCUSSION}

In this report, we analyzed a set of patients treated with sorafenib after recurrence to curative surgery and found an increase in FGF19 copy number alterations in pretreatment tissues from CR cases compared to those from non-CR. The median time from surgery to initiation of sorafenib therapy of CR/PR cases was longer than that of SD/PD cases. Although this result might suggest that duration from surgery to the initiation of sorafenib treatment is related with response to sorafenib, this association might be confounded by the time to recurrence.

Sorafenib, which has been clinically approved for the treatment of patients with advanced HCC [6] [7], is a multi-targeted kinase inhibitor [5]. The genetic alteration in FGFR has been frequently identified as an actionable biomarker [14] [16]. FGFR is not a major target for sorafenib, however, the off-target effect of sorafenib on FGFR might be clinically relevant for HCC tumors with FGF-FGFR alterations. We previously reported that FGF3/FGF4 amplification in HCC might be involved in the response of tumors to sorafenib treatment. However the frequency of $F G F 3 / F G F 4$ amplification was $1-2 \%$ in Japanese HCC cases [11]. In the present study, we found no increases in the copy number of the responders (CR and PR) compared to that of non-responders (SD and PD), however, this was a different sample cohort from that of our previous report [11]. It is possible that differences between the backgrounds of these two cohorts that may have contributed to variances, however, this remains unclear. Potential confounding factors may be attributable to the following: 1) Sample size of this cohort was too small to conclude no amplification was detected in responder cases $(0 / 10) ; 2)$ The criteria for responders in current cohort was different from that of the previous one; in this study a "responder" means CR+PR, whereas a "hyper-responder" means CR. Therefore, we investigated the associations between tumor genetic alterations in FGF-FGFR signals and the efficacy of sorafenib in patients with HCC.

A copy number assay revealed that FGF19 amplification was observed in 4/45 (8.9\%) cases. FGF19 gene amplification has been reported in $7.8 \%$ of liver cancers in the TCGA database (cBioPortal database), consistent with the frequency observed in the present study. In our sample set, the frequency of FGF19 amplification among the $\mathrm{CR}$ cases was significantly higher than that among the other cases $(\mathrm{PR}+\mathrm{SD}+$

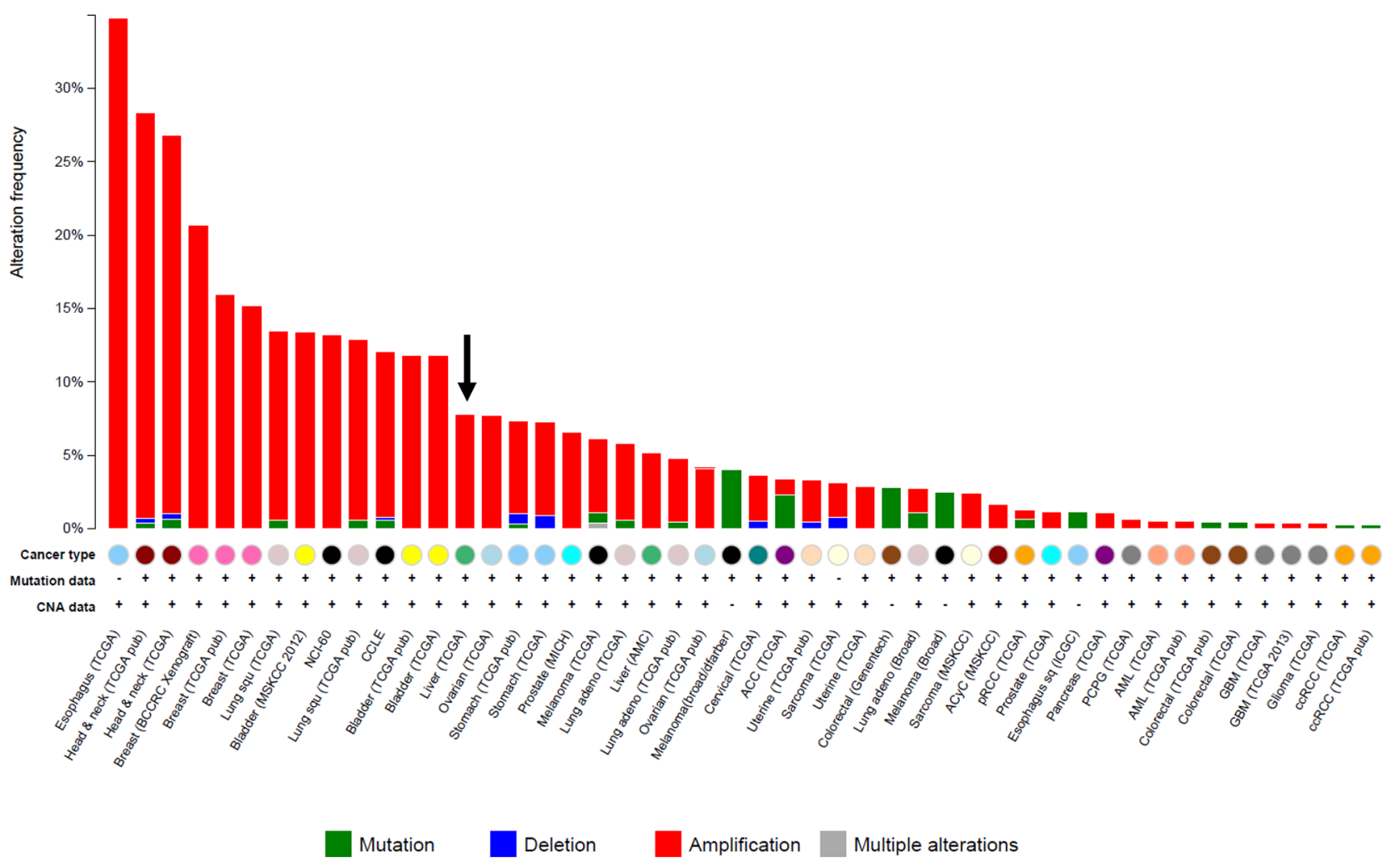

Figure 1: Frequency of FGF19 gene amplification in solid cancers. Gene mutation and copy number alterations in the FGF19 gene were sought using the cBioPortal for Cancer Genomics (http://www.cbioportal.org/public-portal/). The arrow indicates the amplification of FGF19 in HCC. 
PD). FGF19 signaling is mediated via FGFR4, and FGF19-FGFR4 signaling is implicated in hepatocellular tumorigenesis [17] [18]. Molecular studies looking at the role of specific amplicons in HCC identified FGF19 amplification as a potential driver gene of HCC [18]. Preclinical studies showed that the tumors with FGF19 amplification are reportedly associated with a cellular sensitivity to FGFR inhibitors [14]. In the current study, it remains unclear whether increased FGF19 copy numbers correlate with mRNA and protein expression. However, previous reports showed that FGF19 copy number gain correlated well with levels of RNA and protein expression in HCC [18] [19]. Consequently, we speculated that a copy number gain of FGF19 might be a predictor of a response to sorafenib, in addition to $F G F 3$ / FGF4 amplification.

Next-generation sequencing enables a comprehensive analysis of genomic alterations. We focused on FGFR signals and performed targeted DNA sequencing for FGFR1, FGFR2, FGFR3, and FGFR4. In this study, $11.1 \%(5 / 45)$ of the HCC tumors had FGFR mutations, which is slightly higher than the previous report [20]. The frequency of FGFR mutation was not correlated with any clinicopathologic parameters including response to sorafenib. Therefore, we could not establish the significance of the FGFR mutation status and sensitivity to sorafenib. In addition, the biological significance of each mutation remains unclear thus we will need to analyze the functional meaning of each mutation in a future study.

We utilized a retrospective case-control study to explore biomarkers associated with sorafenib response, however, the sample size posed some limitations and there is an inherent susceptibility of selection bias with this type of study design. Therefore, prospective studies will be needed to further evaluate FGF19 copy number gain as a biomarker of response to sorafenib.

In summary, a copy number gain of FGF19 may serve as a potential candidate marker that predicts response to sorafenib, in addition to FGF3/FGF4 amplification. Clinical studies examining a biomarker-driven enriched subpopulation are necessary to determine the efficacy of molecular-targeted treatments with kinase inhibitors. Therefore, the present results suggest new biomarkers for the enrichment of responders to kinase inhibitors.

\section{MATERIALS AND METHODS}

\section{Patients and samples}

Tumor specimens were obtained from a total of 45 HCC patients who had undergone sorafenib treatment at six hospitals between 2004 and 2013. Patients with recurrence after curative surgery were treated with sorafenib. Patients received sorafenib at doses ranging from $100-800 \mathrm{mg} /$ day based on tolerability and drug-related toxicities [21]. Tumor responses were evaluated using the Response Evaluation
Criteria In Solid Tumors (RECIST) ver 1.1 criteria [22]: a complete response (CR) was defined as the disappearance of all measurable and evaluable evidence of disease; a partial response $(\mathrm{PR})$ was defined as a $>30 \%$ decrease in the sum of the longest diameters of target lesions; stable disease (SD) is defined as a less $30 \%$ decrease or less than $20 \%$ increase in the sum of longest diameters; and progressive disease (PD) was indicated by a $>20 \%$ increase in the sum of the longest diameters of target lesions or the appearance of any new lesion. Sixteen of the 22 PD cases showed $>20 \%$ increase in tumor size. Remaining six cases showed bone, lung, and adrenal metastasis. This study was approved by the ethics committee of each institution. All the patients enrolled in the study had provided written informed consent for the use of resected samples. Among the 45 patients, 6 obtained a CR, four had PR, 13 showed SD and 22 showed PD. The study was approved by our Institutional Review Board (Kinki University Faculty of Medicine IRB approval no 25-106) and conformed to the ethical guidelines of the Declaration of Helsinki.

\section{DNA extraction}

All FFPE specimens underwent a histological review, and only those containing sufficient tumor cells (at least 70\% tumor cells) as revealed using hematoxylineosin staining were subjected to DNA extraction. The DNA was purified using an Allprep DNA/RNA FFPE kit (Qiagen, Valencia, CA) according to the manufacturer's instructions. The quality and quantity of the DNA were verified using the NanoDrop 2000 device (Thermo Scientific, Wilmington, DE) and the PicoGreen dsDNA assay kit (Life Technologies, Foster City, CA). The extracted DNA was stored at $-80^{\circ} \mathrm{C}$ until analysis.

\section{DNA sequencing}

Ten nanograms of DNA were used for the multiplex PCR amplification using the Ion AmpliSeq Library kit 2.0 (Life Technologies). We used a custom panel designed for $F G F R 1, F G F R 2, F G F R 3$, and FGFR4 genes. The Ion Xpress Barcode Adapters (Life Technologies) were ligated into the PCR products and were purified with Agencourt AMPure XP beads (Beckman Coulter, Brea, CA). The purified libraries were then pooled and sequenced using an Ion Torrent PGM device (Life Technologies) using the Ion PGM 200 Sequencing kit v2 (Life Technologies) and the Ion 318 v2 Chip kit (Life Technologies). DNA sequencing data were accessed through the Torrent Suite v.4.0 software program. Reads were aligned against the hg19 human reference genome, and variants were called using the variant caller ver. 4.0. Raw variant calls were filtered out using the following annotations: homozygous and heterozygous variants, quality score of $<100$ and depth of coverage $<19$. Germline mutations were excluded using the Human Genetic Variation Database (http://www. genome.med.kyoto-u.ac.jp/SnpDB) [23]. 


\section{Copy number assay}

The copy numbers for $F G F 3, F G F 4, F G F 19$, FGFR1, FGFR2, FGFR3, and FGFR4 were determined using commercially available and predesigned TaqMan Copy Number Assays according to the manufacturer's instructions (Applied Biosystems, Foster City, CA), as described previously [11]. FAM-labeled primers for the target gene and a VIC-labeled primer for TERT as an endogenous control were used for the duplex assay. The primer IDs used for the FGFRs and FGFs were as follows: FGF3, Hs06336027_cn; FGF4, Hs01235235 cn; FGF19, Hs00147838_cn; FGFR1, Hs02164585_cn; FGFR2, Hs05208783 cn; FGFR3, Hs00113109 cn; and FGFR4, Hs01949336_cn. The copy numbers of the target genes were determined by relative quantification using the Copy-Caller-Software, v1.0 (Applied Biosystems). Normal female genomic DNA (Promega; Madison, WI) was used as the normal control (two copies). A cut-off value for copy number gain was set at 5.00 for each gene as described previously [11].

\section{Bioinformatics analysis of genomics of $\mathrm{HCC}$}

To analyze the prevalence of genomic alterations in the FGF19 gene in HCC tissues, the database of the cBioPortal for Cancer Genomics (http:// www.cbioportal. org/public-portal/) was searched. Both copy number variations and gene mutation data were analyzed across cancer types, focusing on HCC. The functional impact of the mutations was assessed based on evolutionary conservation of the affected amino acid in protein homologs, and the predicted functional impact score was assessed using Mutation Assessor (http://mutationassessor.org/).

\section{Statistical analysis}

The Pearson chi-squared test was used to compare the associations between FGF19 copy number alterations and sorafenib efficacy. All the statistical analyses were performed using JMP software (ver. 10; SAS Institute). A $P$ value of $<0.05$ was considered statistically significant.

\section{ACKNOWLEDGMENTS}

We thank the staff of the Life Science Institute of Kinki University for their technical support in performing this study; Mr. Takuya Wada, Mr. Yoshihiro Mine and Mrs. Eiko Honda. We also thank Mr. Yoshimi Hosono, Mrs. Tomoko Miyazaki and Ms. Ayaka Kurumatani for their technical assistance.

\section{CONFLICTS OF INTEREST}

None of the authors have any potential conflicts of interest to report.

\section{FUNDING}

This work was supported by Japan Society for the Promotion of Science (JSPS) KAKENHI Grant Number 24240122.

\section{REFERENCES}

1. Tejeda-Maldonado J, Garcia-Juarez I, Aguirre-Valadez J, Gonzalez-Aguirre A, Vilatoba-Chapa M, ArmengolAlonso A, Escobar-Penagos F, Torre A, Sanchez-Avila JF and Carrillo-Perez DL. Diagnosis and treatment of hepatocellular carcinoma: An update. World J Hepatol. 2015; 7:362-376.

2. Guichard C, Amaddeo G, Imbeaud S, Ladeiro Y, Pelletier L, Maad IB, Calderaro J, Bioulac-Sage P, Letexier M, Degos F, Clement B, Balabaud C, Chevet E, Laurent A, Couchy G, Letouze $\mathrm{E}$, et al. Integrated analysis of somatic mutations and focal copy-number changes identifies key genes and pathways in hepatocellular carcinoma. Nat Genet. 2012; 44:694-698.

3. Revill K, Wang T, Lachenmayer A, Kojima K, Harrington A, Li J, Hoshida Y, Llovet JM and Powers S. Genomewide methylation analysis and epigenetic unmasking identify tumor suppressor genes in hepatocellular carcinoma. Gastroenterology. 2013; 145:1424-1435 e1421-1425.

4. Villanueva A and Llovet JM. Liver cancer in 2013: Mutational landscape of HCC - the end of the beginning. Nat Rev Clin Oncol. 2014; 11:73-74.

5. Wilhelm SM, Carter C, Tang L, Wilkie D, McNabola A, Rong H, Chen C, Zhang X, Vincent P, McHugh M, Cao Y, Shujath J, Gawlak S, Eveleigh D, Rowley B, Liu L, et al. BAY 43-9006 exhibits broad spectrum oral antitumor activity and targets the RAF/MEK/ERK pathway and receptor tyrosine kinases involved in tumor progression and angiogenesis. Cancer Res. 2004; 64:7099-7109.

6. Llovet JM, Ricci S, Mazzaferro V, Hilgard P, Gane E, Blanc JF, de Oliveira AC, Santoro A, Raoul JL, Forner A, Schwartz M, Porta C, Zeuzem S, Bolondi L, Greten TF, Galle PR, et al. Sorafenib in advanced hepatocellular carcinoma. N Engl J Med. 2008; 359:378-390.

7. Cheng AL, Kang YK, Chen Z, Tsao CJ, Qin S, Kim JS, Luo R, Feng J, Ye S, Yang TS, Xu J, Sun Y, Liang H, Liu J, Wang J, Tak WY, et al. Efficacy and safety of sorafenib in patients in the Asia-Pacific region with advanced hepatocellular carcinoma: a phase III randomised, double-blind, placebocontrolled trial. Lancet Oncol. 2009; 10:25-34.

8. Inuzuka T, Nishikawa H, Sekikawa A, Takeda H, Henmi S, Sakamoto A, Saito S, Kita R, Kimura T, Osaki Y and Kudo M. Complete response of advanced hepatocellular carcinoma with multiple lung metastases treated with sorafenib: a case report. Oncology. 2011; 81:152-157.

9. Nakazawa T, Hidaka H, Shibuya A and Koizumi W. Rapid regression of advanced hepatocellular carcinoma associated with elevation of des-gamma-carboxy prothrombin after 
short-term treatment with sorafenib - a report of two cases. Case Rep Oncol. 2010; 3:298-303.

10. So BJ, Bekaii-Saab T, Bloomston MA and Patel T. Complete clinical response of metastatic hepatocellular carcinoma to sorafenib in a patient with hemochromatosis: a case report. J Hematol Oncol. 2008; 1:18.

11. Arao $T$, Ueshima $K$, Matsumoto $K$, Nagai $T$, Kimura $H$, Hagiwara S, Sakurai T, Haji S, Kanazawa A, Hidaka H, Iso Y, Kubota K, Shimada M, Utsunomiya T, Hirooka M, Hiasa Y, et al. FGF3/FGF4 amplification and multiple lung metastases in responders to sorafenib in hepatocellular carcinoma. Hepatology. 2013; 57:1407-1415.

12. Dienstmann R, Rodon J, Prat A, Perez-Garcia J, Adamo B, Felip E, Cortes J, Iafrate AJ, Nuciforo P and Tabernero J. Genomic aberrations in the FGFR pathway: opportunities for targeted therapies in solid tumors. Ann Oncol. 2014; 25:552-563.

13. Weiss J, Sos ML, Seidel D, Peifer M, Zander T, Heuckmann JM, Ullrich RT, Menon R, Maier S, Soltermann A, Moch H, Wagener P, Fischer F, Heynck S, Koker M, Schottle J, et al. Frequent and focal FGFR1 amplification associates with therapeutically tractable FGFR1 dependency in squamous cell lung cancer. Sci Transl Med. 2010; 2:62ra93.

14. Guagnano V, Kauffmann A, Wohrle S, Stamm C, Ito M, Barys L, Pornon A, Yao Y, Li F, Zhang Y, Chen Z, Wilson CJ, Bordas V, Le Douget M, Gaither LA, Borawski J, et al. FGFR genetic alterations predict for sensitivity to NVPBGJ398, a selective pan-FGFR inhibitor. Cancer Discov. 2012; 2:1118-1133.

15. Kumar R, Crouthamel MC, Rominger DH, Gontarek RR, Tummino PJ, Levin RA and King AG. Myelosuppression and kinase selectivity of multikinase angiogenesis inhibitors. Br J Cancer. 2009; 101:1717-1723.

16. Dieci MV, Arnedos M, Andre F and Soria JC. Fibroblast growth factor receptor inhibitors as a cancer treatment: from a biologic rationale to medical perspectives. Cancer Discov. 2013; 3:264-279.
17. Wu X, Ge H, Lemon B, Vonderfecht S, Weiszmann J, Hecht R, Gupte J, Hager T, Wang Z, Lindberg R and Li Y. FGF19induced hepatocyte proliferation is mediated through FGFR4 activation. J Biol Chem. 2010; 285:5165-5170.

18. Sawey ET, Chanrion M, Cai C, Wu G, Zhang J, Zender L, Zhao A, Busuttil RW, Yee H, Stein L, French DM, Finn RS, Lowe SW and Powers S. Identification of a therapeutic strategy targeting amplified FGF19 in liver cancer by Oncogenomic screening. Cancer Cell. 2011; 19:347-358.

19. Miura S, Mitsuhashi N, Shimizu H, Kimura F, Yoshidome H, Otsuka M, Kato A, Shida T, Okamura D and Miyazaki M. Fibroblast growth factor 19 expression correlates with tumor progression and poorer prognosis of hepatocellular carcinoma. BMC Cancer. 2012; 12:56.

20. Ahn SM, Jang SJ, Shim JH, Kim D, Hong SM, Sung CO, Baek D, Haq F, Ansari AA, Lee SY, Chun SM, Choi S, Choi HJ, Kim J, Kim S, Hwang S, et al. Genomic portrait of resectable hepatocellular carcinomas: implications of RB1 and FGF19 aberrations for patient stratification. Hepatology. 2014; 60:1972-1982.

21. Takeda H, Nishikawa H, Osaki Y, Tsuchiya K, Joko K, Ogawa C, Taniguchi H, Orito E, Uchida Y and Izumi N. Clinical features associated with radiological response to sorafenib in unresectable hepatocellular carcinoma: a large multicenter study in Japan. Liver Int. 2015; 35:1581-1589.

22. Eisenhauer EA, Therasse P, Bogaerts J, Schwartz LH, Sargent D, Ford R, Dancey J, Arbuck S, Gwyther S, Mooney M, Rubinstein L, Shankar L, Dodd L, Kaplan R, Lacombe D and Verweij J. New response evaluation criteria in solid tumours: revised RECIST guideline (version 1.1). Eur J Cancer. 2009; 45:228-247.

23. Narahara M, Higasa K, Nakamura S, Tabara Y, Kawaguchi T, Ishii M, Matsubara K, Matsuda F and Yamada R. Largescale East-Asian eQTL mapping reveals novel candidate genes for LD mapping and the genomic landscape of transcriptional effects of sequence variants. PLoS One. 2014; 9:e100924. 